\title{
Is SARS-CoV-2 Spike glycoprotein impairing macrophage polarization via $\alpha 7$-nicotinic acetylcholine receptors?
}

\author{
Tanmay Saraiya, Dimitris Labrou, Konstantinos Farsalinos ${ }^{1}$, Konstantinos Poulas $^{2}$ \\ 1 University of West Attica \\ 2 University of Patras
}

Funding: The author(s) received no specific funding for this work.

Potential competing interests: The author(s) declared that no potential competing interests exist.

\section{Abstract}

The innate immune cells play an important role in handling early infections, and can eliminate them completely up to a certain threshold. Beyond that threshold they take up their role in "The Resolution of Inflammation". The recognition of the SARS-CoV-2 antigen triggers an eicosanoid storm and initiates a robust inflammatory response. This establishes a positive feedback loop which develops into a sustained cytokine storm which interferes with the activation of adaptive immune cells. The mechanism of this interaction, and hence the pathogenesis of the virus with the immune system, is yet to be determined. In silico studies predict a direct SARS-CoV-2 spike glycoprotein interaction with nicotinic acetylcholine receptors, which could impair macrophage function and initiate the cascade of events described above. We here, add to the hypothesis that immune dysregulation can be caused by the interaction of the SARS-CoV-2 spike glycoprotein via a cryptic epitope with the $\alpha 7-n A C h R$ in Type-1 macrophages, discuss its implications for the treatment of COVID-19 patients, and present better prospects for the design and dissemination of more effective vaccines and their importance.

\section{Introduction}

The high expression levels of the angiotensin converting enzyme 2 (ACE2) receptor on lung epithelial cells ${ }^{[1][2]}$ explains why the lung is severely affected by COVID-19, but the infection of resident alveolar macrophages seems counterintuitive, as their expression of the ACE2 receptor is fairly limited [3][4]. Macrophages are an attractive target due to their ability to cause type 1 interferon (IFN) disruption via the activation of various types of pattern recognition receptors (PRRs) they express ${ }^{[5]}$. In theory, the phagocytosis of infected cells by resident macrophages exposes them to the virus ${ }^{5][6]}$. The activation of PRRs in macrophages and other innate cells triggers the release of CD14+ monocytes from the bone marrow into the bloodstream via CCL-2 signaling. They are then induced as resident macrophages at the infection site [5][7]. Monocytes, on the other hand, express the ACE2 receptor and are highly susceptible to SARS-CoV-2 [8][9]. They play a fundamental role in generating an adaptive immune response, as tissue-resident macrophages are poor antigen presenting cells (APCs) and fail to migrate to regional lymph nodes ${ }^{[10]}$. 
The local concentration of proinflammatory cytokines is proportional to the rate of signaling to the bone marrow for the release of monocytes, which further contributes to the production of local proinflammatory cytokines, thereby establishing a positive feedback system. This is naturally regulated by polarization of resident M1 macrophages to the M2 phenotype, which mediate the anti-inflammatory effects and initiate the recovery process ${ }^{[11]}$. By keeping the macrophage population balanced towards the M1 phenotype in the microenvironment, the virus ensures an increase in the concentration of proinflammatory cytokines while avoiding the hindrance caused by anti-inflammatory cytokines ${ }^{[12]}$. This initiates an eicosanoid storm and then a subsequent cytokine storm with increased expression of tumor necrosis factor- $\alpha$ (TNF- $\alpha$ ), as is seen in COVID-19 cases ${ }^{[13][14] .}$

Upon the initiation of the adaptive immune response, neural pathways play a major role in transmitting early signals to and fro, between the infection site and the central nervous system (CNS). The inflammatory reflex is an example; it is responsible for priming the spleen for the adaptive immune response ${ }^{[15][16]}$. At the end of the inflammatory reflex mechanism the indirect activation of $\alpha 7$ - nicotinic Acetylcholine receptor ( $\alpha 7-n A C h R)$ via Acetylcholine produced by splenic T cells inhibits splenic macrophages from expressing proinflammatory cytokines. This is one of the functions associated with M2 macrophages. Whether polarization is triggered by the activation of this receptor or is only a small part of the grand polarization scheme awaits investigation, but it plays a role in the dysfunction of innate immune cells ${ }^{[17]}$.

$\alpha 7-n A C h R$ is the nicotinic type of cholinergic receptors that are activated by the action of Acetylcholine, the neurotransmitter used by the Parasympathetic Nervous System (PSNS) ${ }^{[18]}$. The direct interaction of the virus with macrophages hints at the involvement of $\alpha 7-n A C h R$ in the pathogenesis of COVID-19, indicating a dysregulation of the polarization mechanism, which is an explanation for the dysfunction observed in the innate immune response to this virus ${ }^{[19]}$.

\section{The Misdirection}

SARS-CoV-2 has one of the largest known viral genomes (approximately 2-3 times the average viral length) and features exonuclease activity with proofreading capabilities via nsp14 (ExoN) ${ }^{[20]}$. The term "error catastrophe" has not been defined for this viral candidate. It is adapting well, and a good example here would be the mutations seen in the S1 subunit of the spike protein enabling it to have better binding affinities for the ACE2 receptor as compared to the initial SARS-CoV2 strains while maintaining $~ 76 \%$ similarity with the S1 subunit of SARS-CoV [21][22]. The S2 subunit is even more highly conserved with one striking feature, the biosynthesis of a furin cleavage site that aids the transmissibility of the virus. This is particularly intriguing because the cleavage of S1/S2 subunits is not even necessary for its biosynthesis ${ }^{[22]}$. So why did that happen? Why increase the chances for cleavage of a site that is not necessary in the first place? This suggests that the $\mathrm{S} 1$ residue still has a few tricks up its sleeve and these come into effect post binding with the transmembrane pentameric glycoprotein receptor ACE2.

The epitope (aa 375-390) belongs to a domain of the S1 subunit and it is highly conserved among the global mutations in

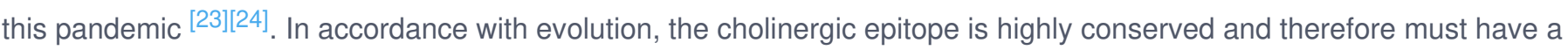


function. Upon initial assessments, In silico models predicted a direct interaction with innate immune cells expressing the ACE2 receptor and $\alpha 7-n A C h R$, based on which further In-vitro experiments are ongoing ${ }^{[25][26]}$.

The SARS-CoV antibody, CR3022 that neutralized its cholinergic epitope also interacts with high affinity to SARS-CoV-2 and is accessible only in the open configuration of the trimeric spike receptor binding domain (RBD) [24][27]. CR3022 in combination with CR3014, that neutralized the spike RBD, was successful in completely neutralizing SARS-CoV but failed to do so in this case due to the mutations observed between the S1 regions of SARS-CoV and SARS-CoV-2 [28]. This theory is supported by the rare SARS-CoV-2 antibody COVA1-16 that is also encoded to this cholinergic epitope [29].

\section{The antibody conundrum}

The viral load a COVID-19 host faces is comparable to that of a simultaneous attack by multiple viruses due to the mutations observed in the spike protein. This is why one antibody may work against a few strands but is ineffective in neutralizing the others, thereby reducing the overall efficacy of our vaccines. There is no generic antibody capable of neutralizing all of the mutated spike proteins, yet the only way to develop long-term protective immunity against this virus is with an antibody. The human body is capable of generating a diverse antibody population required to handle immense viral loads, provided that the immune system is intact. Kaneko et al. (2020) observed low but diverse antibody production in COVID-19 cases ${ }^{[30]}$. This can be explained as with the onset of SARS-CoV-2 infection, the immune system starts to dysregulate leading to B-cell lymphopenia. An increase in proinflammatory cytokines is accompanied by an increase in TNF- $\alpha$, which inhibits the differentiation of active CD4+ T cells into BCL6+ GC-T $T_{F H}$ cells (that aid with B cell antibody production) subsequently resulting in a loss of germinal centers. Naturally, the antibodies formed are insufficient, leaving us susceptible to reinfection, assuming that the patient has recovered from the first infection [30].

\section{Implications for Clinical therapy}

An antibody targeting an epitope that is conserved across all mutations worldwide that interacts with immune cells has major benefits. Neutralizing this epitope would handicap the ability of the virus to cause dysfunction, but this is only a part of the $\mathrm{S} 1$ residue. The main virion continues its infection cycle.

In severe COVID-19 cases an additional stimulation of $\alpha 7-n A C h R$ would be essential to restore the integrity of the immune system. A combination of $\alpha 7-n A C h R$ agonists along with the proposed antibody will definitely help regulate the proinflammatory cytokine concentrations, but whether that alone would suffice to restore the ability of the body to generate sufficient quantities of antibodies for a long term immunity requires further evaluation [31][32][33].

\section{Conclusion}

COVID-19 shares its clinical features with autoimmune and inflammatory diseases that have imbalanced M1/M2 ratios, and cholinergic activation in those conditions has shown alleviating effects. Due to these similarities, researchers have 
focused on deactivating certain pathways and cytokines, but the results are inconclusive, mainly because they interfere with a recovery process that is essential at some point during the infection cycle. Dysregulation of these pathways cause downstream effects that eventually benefit the virus or worsen the condition of the patient. Treatment should be designed in such a way that it does not interfere with natural processes while still effectively terminating the viral infection.

The evolution of SARS-CoV-2 is mediated through variants. They adapt to evade the immune response and this is seen as the varaints with mutations in the spike protein have better adhesion capabilities and therefore higher transmission rates ${ }^{[34][35]}$. This had forced us to upgrade our vaccines from antibody cocktails to mRNA and adenoviral vectors that encoded the most studied protein, i.e. spike. The issue with mRNA and adenoviral vectored vaccines is that they all encode the spike protein, which is the hidden mastermind behind a SARS-CoV-2 infection. It creates a distraction by continuously evolving its RBD, while in essence, the disease is caused by disrupting the immune response via the cholinergic epitope. Also, note that it is possible that the vaccines itself might facilitate recombinant mutations. For example, if people in India are inoculated with vaccines that encode the spike of a variant from outside India, say UK, the local strains are now exposed to strains from the UK.

So in the long term perspective the efficiencies of our current vaccines will keep degrading as the virus mutates till we exhaust our capacity to practically produce the booster shots on a global scale ${ }^{[36]}$.

Our efforts to develop vaccines would be better suited if we redirect our attention to this epitope and figure out the exact function of its interaction with our body, more specifically how it affects the macrophage polarization scheme.

\section{References}

1. ^Hong Peng Jia, Dwight C. Look, Lei Shi, Melissa Hickey, et al. (2005).ACE2 Receptor Expression and Severe Acute Respiratory Syndrome Coronavirus Infection Depend on Differentiation of Human Airway Epithelia. JVI, vol. 79 (23), 14614-14621. doi:10.1128/jvi.79.23.14614-14621.2005.

2. ^I Hamming, W Timens, MLC Bulthuis, AT Lely, et al. (2004).Tissue distribution of ACE2 protein, the functional receptor for SARS coronavirus. A first step in understanding SARS pathogenesis. J. Pathol., vol. 203 (2), $631-637$. doi:10.1002/path.1570.

3. `Koichi Yuki, Miho Fujiogi, Sophia Koutsogiannaki. (2020). COVID-19 pathophysiology: A review. Clinical Immunology, vol. 215 , 108427. doi:10.1016/j.clim.2020.108427.

4. ' Yao, X H et al.. (2020). Zhonghua Bing Li Xue Za Zhi. Chinese journal of pathology, vol. 49,5: 411-417.

5. a, b, c Abdollah Jafarzadeh, Prashant Chauhan, Bhaskar Saha, Sara Jafarzadeh, et al. (2020). Contribution of monocytes and macrophages to the local tissue inflammation and cytokine storm in COVID-19: Lessons from SARS and MERS, and potential therapeutic interventions. Life Sciences, vol. 257 , 118102. doi:10.1016/j.Ifs.2020.118102.

6. `Ekaterina Nikitina, Irina Larionova, Evgeniy Choinzonov, Julia Kzhyshkowska. (2018). Monocytes and Macrophages as Viral Targets and Reservoirs. IJMS, vol. 19 (9), 2821. doi:10.3390/ijms19092821. 
7. ^Amanda M. Guth, William J. Janssen, Catharine M. Bosio, Erika C. Crouch, et al. (2009) Lung environment determines unique phenotype of alveolar macrophages. American Journal of Physiology-Lung Cellular and Molecular Physiology, vol. 296 (6), L936-L946. doi:10.1152/ajplung.90625.2008.

8. 'Dan Zhang, Rui Guo, Lei Lei, Hongjuan Liu, et al. (2020).COVID-19 infection induces readily detectable morphological and inflammation-related phenotypic changes in peripheral blood monocytes, the severity of which correlate with patient outcome. doi:10.1101/2020.03.24.20042655.

9. ' Li Liu, Qiang Wei, Qingqing Lin, Jun Fang, et al. (2019).Anti-spike lgG causes severe acute lung injury by skewing macrophage responses during acute SARS-CoV infection. doi:10.1172/jci.insight.123158.

10. 'David A. Hume. (2008). Macrophages as APC and the Dendritic Cell Myth. J Immunol, vol. 181 (9), 5829-5835. doi:10.4049/jimmunol.181.9.5829.

11. ^Almudena Ortega-Gómez, Mauro Perretti, Oliver Soehnlein. (2013). Resolution of inflammation: an integrated view. EMBO Mol Med, vol. 5 (5), 661-674. doi:10.1002/emmm.201202382.

12. 'Daniel W. Lee, Rebecca Gardner, David L. Porter, Chrystal U. Louis, et al. (2014).Current concepts in the diagnosis and management of cytokine release syndrome. doi:10.1182/blood-2014-05-552729.

13. 'Dipak Panigrahy, Molly M. Gilligan, Sui Huang, Allison Gartung, et al. (2020).Inflammation resolution: a dual-pronged approach to averting cytokine storms in COVID-19?. Cancer Metastasis Rev, vol. 39 (2), 337-340. doi:10.1007/s10555020-09889-4.

14. ^Víctor J. Costela-Ruiz, Rebeca Illescas-Montes, Jose M. Puerta-Puerta, Concepción Ruiz, et al. (2020).SARS-CoV-2 infection: The role of cytokines in COVID-19 disease. Cytokine \& Growth Factor Reviews, vol. 54 , 62-75. doi:10.1016/j.cytogfr.2020.06.001.

15. ' Peder S. Olofsson, Mauricio Rosas-Ballina, Yaakov A. Levine, Kevin J. Tracey. (2012).Rethinking inflammation: neural circuits in the regulation of immunity. doi:10.1111/j.1600-065x.2012.01138.x.

16. `Sangeeta S. Chavan, Valentin A. Pavlov, Kevin J. Tracey. (2017).Mechanisms and Therapeutic Relevance of Neuroimmune Communication. Immunity, vol. 46 (6), 927-942. doi:10.1016/j.immuni.2017.06.008.

17. ^ Carlos A. Báez-Pagán, Manuel Delgado-Vélez, José A. Lasalde-Dominicci. (2015).Activation of the Macrophage $\alpha 7$ Nicotinic Acetylcholine Receptor and Control of Inflammation. J Neuroimmune Pharmacol, vol. 10 (3), 468-476. doi:10.1007/s11481-015-9601-5.

18. 'Prashant Tiwari, Shubhangi Dwivedi, Mukesh Pratap Singh, Rahul Mishra, et al. (2013).Basic and modern concepts on cholinergic receptor: A review. Asian Pacific Journal of Tropical Disease, vol. 3 (5), 413-420. doi:10.1016/s22221808(13)60094-8.

19. ^Alexander P. Horkowitz, Ashley V. Schwartz, Carlos A. Alvarez, Edgar B. Herrera, et al. (2020).Acetylcholine regulates pulmonary inflammation and facilitates the transition from active immunity to tissue repair during respiratory viral infection. doi:10.1101/2020.07.02.184226.

20. 'Maria Romano, Alessia Ruggiero, Flavia Squeglia, Giovanni Maga, et al. (2020).A Structural View of SARS-CoV-2 RNA Replication Machinery: RNA Synthesis, Proofreading and Final Capping. Cells, vol. 9 (5), 1267. doi:10.3390/cells9051267.

21. 'Seiya Ozono, Yanzhao Zhang, Hirotaka Ode, Kaori Sano, et al. (2021).SARS-CoV-2 D614G spike mutation 
increases entry efficiency with enhanced ACE2-binding affinity. Nat Commun, vol. 12 (1). doi:10.1038/s41467-02121118-2.

22. ${ }^{a, b}$ Alexandra C. Walls, Young-Jun Park, M. Alejandra Tortorici, Abigail Wall, et al. (2020). Structure, Function, and Antigenicity of the SARS-CoV-2 Spike Glycoprotein. Cell, vol. 181 (2), 281-292.e6. doi:10.1016/j.cell.2020.02.058.

23. `GSAID data on Los Alamos National Laboratory, 2021. Spike Mutation table. https://cov.lanl.gov/components/sequence/COV/int_sites_tbls.comp?t=1\&l=(accessed 29 March 2021).

24. ${ }^{a, b}$ Meng Yuan, Nicholas C. Wu, Xueyong Zhu, Chang-Chun D. Lee, et al. (2020) A highly conserved cryptic epitope in the receptor binding domains of SARS-CoV-2 and SARS-COV. Science, vol. 368 (6491), 630-633. doi:10.1126/science.abb7269.

25. ^George Lagoumintzis, Christos T. Chasapis, Nikolaos Alexandris, Dimitrios Kouretas, et al. (2021).Nicotinic cholinergic system and COVID-19: In silico identification of interactions between $\alpha 7$ nicotinic acetylcholine receptor and the cryptic epitopes of SARS-Co-V and SARS-CoV-2 Spike glycoproteins. Food and Chemical Toxicology, vol. 149 , 112009. doi:10.1016/j.fct.2021.112009.

26. 'Konstantinos Farsalinos, Elias Eliopoulos, Demetres D. Leonidas, Georgios E. Papadopoulos, et al. (2020).Nicotinic Cholinergic System and COVID-19: In Silico Identification of an Interaction between SARS-CoV-2 and Nicotinic Receptors with Potential Therapeutic Targeting Implications. IJMS, vol. 21 (16), 5807. doi:10.3390/ijms21165807.

27. 'M. Gordon Joyce, Rajeshwer S. Sankhala, Wei-Hung Chen, Misook Choe, et al. (2020).A Cryptic Site of Vulnerability on the Receptor Binding Domain of the SARS-CoV-2 Spike Glycoprotein. doi:10.1101/2020.03.15.992883.

28. `Jan ter Meulen, Edward N van den Brink, Leo L. M Poon, Wilfred E Marissen, et al. (2006).Human Monoclonal Antibody Combination against SARS Coronavirus: Synergy and Coverage of Escape Mutants. PLoS Med, vol. 3 (7), e237. doi:10.1371/journal.pmed.0030237.

29. ^Hejun Liu, Nicholas C. Wu, Meng Yuan, Sandhya Bangaru, et al. (2020).Cross-neutralization of a SARS-CoV-2 antibody to a functionally conserved site is mediated by avidity. doi:10.1101/2020.08.02.233536.

30. a, b Naoki Kaneko, Hsiao-Hsuan Kuo, Julie Boucau, Jocelyn R. Farmer, et al. (2020).Loss of Bcl-6-Expressing T Follicular Helper Cells and Germinal Centers in COVID-19. Cell, vol. 183 (1), 143-157.e13. doi:10.1016/j.cell.2020.08.025.

31. ^Ravikumar A. Sitapara, Alex G. Gauthier, Sergio I. Valdés-Ferrer, Mosi Lin, et al. (2020).The $\alpha 7$ nicotinic acetylcholine receptor agonist, GTS-21, attenuates hyperoxia-induced acute inflammatory lung injury by alleviating the accumulation of HMGB1 in the airways and the circulation. Mol Med, vol. 26 (1). doi:10.1186/s10020-020-00177-z.

32. 'Ulf Andersson. (2020). The cholinergic anti-inflammatory pathway alleviates acute lung injury. Mol Med, vol. 26 (1). doi:10.1186/s10020-020-00184-0.

33. 'Nikolaos Alexandris, George Lagoumintzis, Christos T. Chasapis, Demetres D. Leonidas, et al. (2021).Nicotinic cholinergic system and COVID-19: In silico evaluation of nicotinic acetylcholine receptor agonists as potential therapeutic interventions. Toxicology Reports, vol. 8 , 73-83. doi:10.1016/j.toxrep.2020.12.013.

34. ' CDC, 2021. Variant tracking. https://www.cdc.gov/coronavirus/2019-ncov/science/science-briefs/scientific-briefemerging-variants.html?CDC_AA_refVal=https\%3A\%2F\%2Fwww.cdc.gov\%2Fcoronavirus\%2F2019ncov\%2Fmore\%2Fscience-and-research\%2Fscientific-brief-emerging-variants.html (accessed 29 March 2021). 
35. ' Qianqian Li, Jiajing Wu, Jianhui Nie, Li Zhang, et al. (2020). The Impact of Mutations in SARS-CoV-2 Spike on Viral Infectivity and Antigenicity. Cell, vol. 182 (5), 1284-1294.e9. doi:10.1016/j.cell.2020.07.012.

36. 'Chloe Rees-Spear, Luke Muir, Sarah A. Griffith, Judith Heaney, et al. (2021). The effect of spike mutations on SARSCoV-2 neutralization. Cell Reports, vol. 34 (12), 108890. doi:10.1016/j.celrep.2021.108890. 